\title{
Looking, and thinking, about your genome like real estate: location, location, location
}

\author{
David F. Albertini ${ }^{1}$
}

Published online: 26 March 2015

(C) Springer Science+Business Media New York 2015

Inheriting property from your forbears, much like their genomic counterparts, ends up being for better or worse. Property values will in the end be determined by market conditions, and of course the importance of location-as the adage goescannot be underestimated. That any metaphor about real estate would permeate into the lingo of genomics would have to be considered as bordering the absurd given double helix thought modality bestowed upon us by the likes of Watson and Crick. But, with advances of the past decade, our genomes have emerged as four-dimensional landscapes operationally well in excess of base pairs, promoters, introns and their attendant nucleosomes presently evoking a level of complexity best described as geographical or topological-hence the real estate idea.

Such a conceptualization is buttressed at one level on the principles of epigenetics. Gender-specific modifications in DNA methylation are inheritable as are the metabolic accoutrements that micromanage the histone code of protein posttranslational adjustments, upon whose shoulders sit the genes that will or will not be permitted to speak at appointed stages of development. In the field of reproductive medicine, the ways we think about the origins of disease are in transition and it is our hope that this issue of JARG will bring into

Capsule The greater resolution afforded by the new genetic technologies is deepening and refining our understanding of the origins of disease as related to fertility. However, our perspectives remain rudimentary and are unable to discern the geographical basis of genome remodeling and function during the most critical times of human developmentgametogenesis and preimplantation embryogenesis.

David F. Albertini

dalbertini@kumc.edu

1 University of Kansas Medical Center, Kansas, KS, USA perspective some of the formative technological advances that are reshaping our understanding of the causes of infertility.

Featured on our cover this month is an example of the more customary display of our genomes-the ideogram-that is replacing karyotypes of old owing to the suffusion of information gained from molecular genetics. Just as mapmaking was used to guide and inform key steps in the advancement of civilization, so too do planar representations offer a framework to explore and understand the human genome. Beyond the detail rendered for individual chromosomes, mapping loci with respect to their proximities has been a time-honored paradigm for probing the collaborative and/or collusional interactions between gene clusters believed to underscore complex traits or disease states deriving from neighborly crosstalk within or between chromosomes. Using ideograms to explore disease origins Butler and colleagues review this approach in this issue of JARG with respect to possible linkages between obesity and reproductive disorders (Clinically relevant known and candidate genes for obesity and their overlap with human infertility and reproduction, 10.1007/s10815-014-0411-0).

Together, new approaches such as karyomapping (reviewed last month), ideograms, and polymorphism analyses are enhancing our knowledge base of the human genome generally and specifically as it pertains to diseases of the reproductive tract. In this light, the paper by $\mathrm{Wu}$ and colleagues reviews and offers a meta-analysis in the context of genetic polymorphisms placing patients at risk of developing PCOS. But rather than the usual set of suspects, potential roles for inflammatory mediators are once again brought to light as players in the larger scheme of reproductive diseases (Associations between $T N F-\alpha$ and interleukin gene polymorphisms with polycystic ovary syndrome risk: a systematic review and meta-analysis, 10.1007/s10815-015-0449-7). However, these perspectives remain largely two-dimensional and only begin 
to address larger domains of nuclear geography that will embrace principles of time and three-dimensional space in the future.

Broadly speaking, location works as a reference point with meaning for the disposition of chromosomes as long as fiduciary marks can be defined and ascribed some sort of operational task. Take for example the nucleolus. This is perhaps the first and foremost geographical landmark in eukaryotic nuclei that we all come to appreciate as the ribosome factory. Now that the nucleolus has been dissected in genomic, proteomic, and epigenetic terms, it comes as no surprise to learn of the many other functions subserved by this organelle including regulation of cell cycle progression, RNA processing beyond the scope of ribosomes, and as a warehouse for many gene products requiring sequestration to avoid an untimely demise or and out-of-context post-translational modification. In this regard, it is curious that as our understanding of the nucleolus has been enriched, the obvious dynamic alterations encountered in human embryos during the earliest stages of development have somehow escaped the attention of time-lapse imaging enthusiasts, despite the emphasis that is being placed on cell cycle kinetics as a biomarker of embryo quality. Here enters an intriguing report in this month's JARG by Chamayou and collaborators (Oocyte vitrification modifies nucleolar remodeling and zygote kinetics-a sibling study, $10.1007 / \mathrm{s} 10815-015-0446)$. Studies of this kind will continue to inform ongoing deliberations as to the putative merits of embryo selection technologies and highlight the fact many fundamental attributes of oocytes that are drawn upon to initiate development may be severely impacted by vitrification.

And last, but not least, we encourage our readership to have a close look at the opinion article by Dale and colleagues entitled Trends, Fads and ART! (10.1007/s10815-014-0420 ). With the extreme emphasis that has been placed on embryo selection since the inception of human ARTs, these authors offer what may best be described as a case of missing identity when it comes to what the focus of our efforts should be to achieve optimal chances to pregnancy and healthy offspring. From the misconceptions upon which our framework for quality control is founded, to the fast pace at which ARTs are adopted with seemingly clinical value added, the case is made that what in the end will result in ART success is not a matter of identifying the "best" embryo but rather will be an genuine effort to preserve or manage the integrity of the gametes from which embryos will be derived. We welcome comments from our readership towards this end as completion of the fourth decade of ARTs fast approaches. 